\title{
Sum-frequency excitation of coherent magnons
}

\author{
Dominik M. Juraschek $\odot,{ }^{*}, \dagger$ Derek S. Wang $\odot,{ }^{*}$ and Prineha Narang $\odot *$ \\ Harvard John A. Paulson School of Engineering and Applied Sciences, Harvard University, Cambridge, Massachusetts 02138, USA
}

(Received 2 December 2020; accepted 3 February 2021; published 4 March 2021)

\begin{abstract}
Coherent excitation of magnons is conventionally achieved through Raman scattering processes, in which the difference-frequency components of the driving field are resonant with the magnon energy. Here, we describe mechanisms by which the sum-frequency components of the driving field can be used to coherently excite magnons through two-particle absorption processes. We use the Landau-Lifshitz-Gilbert formalism to compare the spin-precession amplitudes that different types of impulsive stimulated and ionic Raman scattering processes and their sum-frequency counterparts induce in an antiferromagnetic model system. We show that sum-frequency mechanisms enabled by linearly polarized driving fields yield excitation efficiencies comparable or larger than established Raman techniques, while elliptical polarizations produce only weak and circular polarizations no sum-frequency components at all. The mechanisms presented here complete the map for dynamical spin control by means of Raman-type processes.
\end{abstract}

DOI: 10.1103/PhysRevB.103.094407

Ultrashort laser pulses are able to generate coherent quasiparticle excitations and induce macroscopic amplitudes in the normal mode coordinates, such as atomic motion for phonons and spin precession for magnons. The most prominent excitation mechanisms are Raman processes: impulsive stimulated Raman scattering, in which photons from an ultrashort laser pulse scatter with a Raman-active quasiparticle, and ionic Raman scattering, in which photons from an ultrashort pulse initially excite infrared-active phonons coherently, which then in turn scatter with a Raman-active quasiparticle. Impulsive stimulated Raman scattering is an established means of excitation for Raman-active phonons [1,2] and magnons [3-11]. Ionic Raman scattering in turn, predicted 50 years ago [12-14], has been demonstrated and described only in recent years for Raman-active phonons [15-18] and magnons [19-21] due to the development of powerful terahertz and midinfrared sources.

Phenomenologically, these Raman processes are described by an $A_{\mathrm{R}} A_{\mathrm{D}}^{2}$ interaction term in the free energy, where $A_{\mathrm{R}}$ is the amplitude of the normal mode coordinate of the Ramanactive quasiparticle and $A_{\mathrm{D}}$ is the amplitude of the driving field. In the case of impulsive stimulated Raman scattering, $A_{\mathrm{D}}$ corresponds to the electric field amplitude of the laser pulse, and in the case of ionic Raman scattering, $A_{\mathrm{D}}$ is the vibrational amplitude of the coherent infrared-active phonon. If the driving field follows a sinusoidal shape $A_{\mathrm{D}} \sim$

\footnotetext{
* D.M.J. and D.S.W. authors contributed equally to this work.

$\dagger$ djuraschek@seas.harvard.edu

"prineha@seas.harvard.edu
}

Published by the American Physical Society under the terms of the Creative Commons Attribution 4.0 International license. Further distribution of this work must maintain attribution to the author(s) and the published article's title, journal citation, and DOI. $\sin \left(\omega_{0} t\right)$ with center frequency $\omega_{0}$, then the force acting on the Raman-active quasiparticle, $A_{\mathrm{D}}^{2} \sim 1-2 \cos \left(2 \omega_{0} t\right)$, produces a static term and one oscillating at double the frequency. For finite linewidths, these terms consist of difference- and sumfrequency components, $\omega_{1}-\omega_{2}$ and $\omega_{1}+\omega_{2}$, respectively. In conventional Raman scattering processes, the differencefrequency components of the driving field are resonant with the frequency of the Raman-active quasiparticle, as illustrated in Figs. 1(a) and 1(b). Very recently, excitations of coherent Raman-active phonons through sum-frequency components have been achieved, in which the Raman-active phonon absorbs two photons [22-25] or two infrared-active phonons [23-28]. In contrast, both experimental and theoretical demonstrations are still missing for the excitation of magnons through sum-frequency processes.

In this study, we develop a phenomenological description of the sum-frequency counterparts [Figs. 1(c) and 1(d)] of impulsive stimulated and ionic Raman scattering for the coherent excitation of magnons in dielectric insulators, building on the formalism developed in Ref. [21]. The sum-frequency description of the scattering process differs conceptually from the Raman scattering processes with phonons [22,23], because angular momentum considerations have to be taken into account. We compare the relative strengths of the difference- and sum-frequency mechanisms for the example of an anisotropic antiferromagnetic Heisenberg model, for which we evaluate the spin-precession amplitudes for different optical and phononic drives. We show that sum-frequency mechanisms enabled by linearly polarized driving fields yield excitation efficiencies comparable to or larger than established Raman techniques for comparable coupling strengths, while elliptical polarization produces only weak and circular polarization no sum-frequency components at all. In addition, the sum-frequency mechanisms benefit from a higher selectivity due to the possibility of resonant tuning and due to the lower energy of the excitation. 
Opto-magnetic
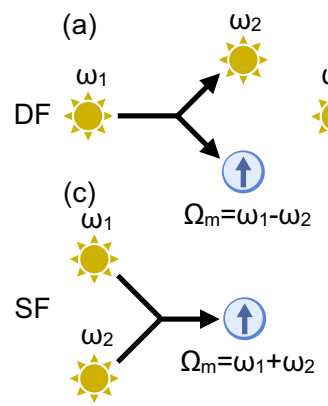

Phono-magnetic

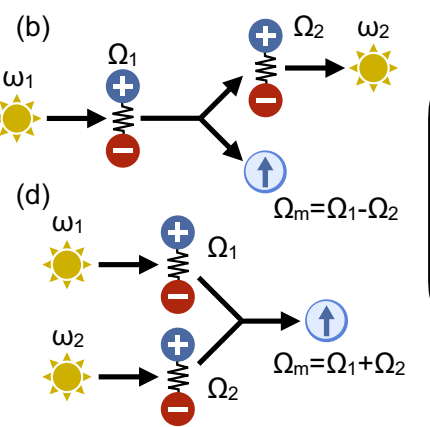

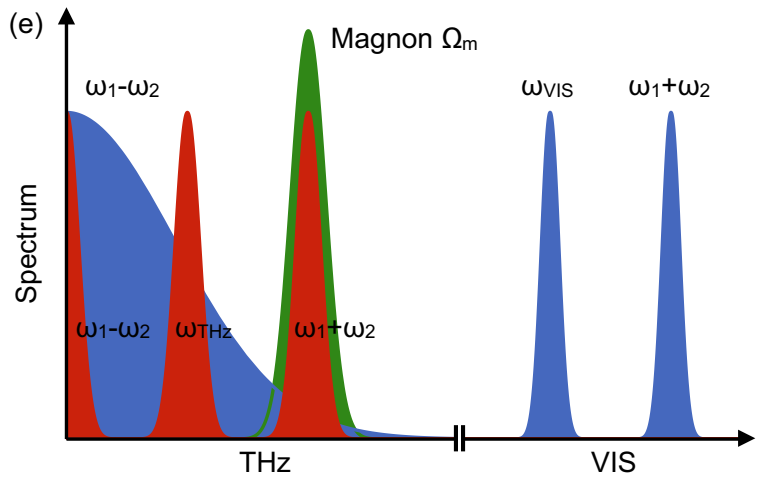

FIG. 1. Fundamental one-magnon scattering processes of photons and coherent infrared-active phonons. (a) Impulsive stimulated Raman scattering, (b) ionic Raman scattering, (c) two-photon absorption, (d) two-phonon absorption. (e) Schematic frequency spectrum of a magnon and optomagnetic drives in the visible and terahertz range. Shown are the frequency of the magnon, $\Omega_{\mathrm{m}}$ (green), the center frequencies of the visible and terahertz pulses, $\omega_{\mathrm{VIS}}$ (blue) and $\omega_{\mathrm{THz}}$ (red), as well as the difference- and sum-frequency components of the respective driving forces, $\omega_{1}-\omega_{2}$ and $\omega_{1}+\omega_{2}$. In this example, the difference-frequency components of the visible-light pulse and the sum-frequency components of the terahertz pulse overlap with the magnon mode and are therefore able to excite it.

\section{THEORY OF OPTICALLY AND PHONON-INDUCED SPIN DYNAMICS}

\section{A. Landau-Lifshitz-Gilbert spin dynamics}

We begin by reviewing the phenomenological theory of spin dynamics in the Landau-Lifshitz-Gilbert formalism. The precession of a spin, $\mathbf{S}_{s}$ with $\left|\mathbf{S}_{s}\right|=1$, follows the equation of motion

$$
\frac{\mathrm{d} \mathbf{S}_{s}}{\mathrm{~d} t}=-\frac{\gamma}{1+\Gamma^{2}}\left[\mathbf{S}_{s} \times \mathbf{B}_{s}^{\mathrm{eff}}-\frac{\Gamma}{\left|\mathbf{S}_{s}\right|} \mathbf{S}_{s} \times\left(\mathbf{S}_{s} \times \mathbf{B}_{s}^{\mathrm{eff}}\right)\right],
$$

where $\gamma=g \mu_{\mathrm{B}} / \hbar, g$ is the material-dependent gyromagnetic ratio, $\mu_{\mathrm{B}}$ is the Bohr magneton, $\hbar$ is the reduced Planck's constant, and $\Gamma$ is a phenomenological damping constant. The effective magnetic field acting on the spin is given by $\mathbf{B}_{s}^{\text {eff }}=(\gamma \hbar)^{-1} \partial H / \partial \mathbf{S}_{s}$, where $H$ is the Hamiltonian of the system. Here, we use a model of a three-dimensional anisotropic Heisenberg antiferromagnet with two spin sublattices, $s \in$ $\{1,2\}$, which interacts with light and coherent phonons,

$$
H=H_{0}+H_{\text {phot }}+H_{\text {phon }} .
$$

The ground state Hamiltonian

$$
H_{0}=J \mathbf{S}_{1} \cdot \mathbf{S}_{2}+\sum_{s=1}^{2}\left[D_{x} S_{s x}^{2}+D_{y} S_{s y}^{2}\right]
$$

consists of a term with antiferromagnetic exchange coupling, $J=6 J_{\mathrm{NN}}>0$, where $J_{\mathrm{NN}}$ is the nearest-neighbor antiferromagnetic exchange coupling, and anisotropy terms along the $x$ and $y$ directions, $D_{x}$ and $D_{y}$, which align the spins along the $z$ direction, as shown schematically in Fig. 2. The studied model and spin lattice is similar to those used to describe the prototypical antiferromagnet nickel oxide (NiO) [29-31], but for demonstration purposes, we use artificial values for the material parameters here that do not reflect the properties of $\mathrm{NiO} . H_{\text {phot }}$ and $H_{\text {phon }}$ describe the interactions of the spins with light and with coherent infrared-active phonons. In the ground state and without external magnetic fields, this model exhibits two magnon modes, one out-of-plane mode with energy $\hbar \Omega_{\mathrm{OP}}=2 \sqrt{J\left(D_{x}+D_{y}\right)}$, and one in-plane mode with energy $\hbar \Omega_{\mathrm{IP}}=2 \sqrt{J D_{y}}$ [31]. The effective magnetic field experienced by the spins in equilibrium, $\mathbf{B}_{s}^{0}$, is given by [30]

$$
\begin{aligned}
\mathbf{B}_{s}^{0} & =\frac{1}{\gamma \hbar} \partial H_{0} / \partial \mathbf{S}_{s} \\
& =\frac{1}{\gamma \hbar}\left[J\left(S_{s x} \hat{x}+S_{s y} \hat{y}+S_{s z} \hat{z}\right)+2\left(D_{x} S_{s x} \hat{x}+D_{y} S_{s y} \hat{y}\right)\right] .
\end{aligned}
$$

In the following sections, we describe the effective magnetic fields exerted on the spins by the electric-field components of a laser pulse (optomagnetic effects) and by coherently excited infrared-active phonons (phonomagnetic effects). We hereby extend the formalism of Ref. [21] to include both difference-frequency components through impulsive stimulated and ionic Raman scattering, as well sumfrequency components through two-photon and two-phonon absorption. The coherent nature of the excitations allows us to treat the fundamentally quantum-mechanical states of coherent phonons and magnons in a semiclassical formalism of vibrations and spin waves [2,32,33]. Both impulsive stimulated and ionic Raman mechanisms here describe one-magnon scattering at the center of the Brillouin zone. For two-magnon

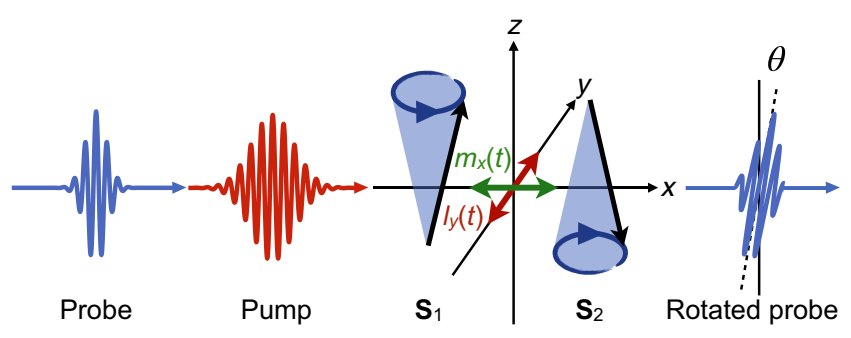

FIG. 2. Schematic of the pump-probe geometry. The spins of the antiferromagnetic model are aligned along the $z$ direction. The in-plane magnon mode excited by the pump pulse in this geometry (either directly, or through coherently excited phonons) distorts the spins antiferromagnetically along the $y$ direction, $l_{y}(t)$, as well as ferromagnetically along the $x$ direction, $m_{x}(t)$. The probe pulse then undergoes a Faraday rotation $\theta(t)$, which follows the oscillation of $m_{x}(t)$. 
scattering processes [34], the scattering conditions in Fig. 1(e) would have to be modified to include two times the magnon frequency. The effective magnetic field picture hereby provides an intuitive description of the underlying physics in the regime of stable magnetic order, where no photo- or phononinduced melting is induced [35-40]. Note that for very short pulses, the diamagnetic response in the effective magnetic field picture may not be entirely accurate anymore and additional diamagnetic effects resulting from a quenching of orbital angular momentum have to be taken into account that may even dominate the response in some cases [41-43]. The scattering process then has to be treated in a fully quantummechanical formalism $[7,44-46]$.

\section{B. Effective optomagnetic fields}

We now derive the effective optomagnetic fields arising from the interaction of the electric-field components of a laser pulse with magnetic order. In conventional descriptions of nonlinear light-matter interactions in the field energy density approach, a time averaging is applied that eliminates fastoscillating components [47-50]. To obtain both differenceand sum-frequency components of the optomagnetic field, we write the field energy density $U$ of a nonabsorbing and linearly polarized material without time averaging as

$$
U=\frac{1}{2} D_{i}(t) E_{i}(t),
$$

where $E_{i}$ is the electric field and $D_{i}=\varepsilon_{0} E_{i}+P_{i}$ the electric displacement field along the spatial coordinate $i$, and $\varepsilon_{0}$ is the vacuum permittivity. The complex spectral decomposition of the electric field reads $E_{i}(t)=\sum_{n} \tilde{E}_{i}\left(\omega_{n}\right) e^{-\mathrm{i} \omega_{n} t}$, where $\tilde{E}_{i}\left(\omega_{n}\right)$ is the amplitude of the electric-field component with frequency $\omega_{n}$, the sum over $n$ runs over all negative and positive frequencies, and $\tilde{E}_{i}\left(\omega_{n}\right)=\tilde{E}_{i}^{*}\left(-\omega_{n}\right)$ to ensure that $E_{i}(t)$ is real. We use the Einstein notation for the summation of indices. The polarization $P_{i}$ up to first order in the electric field is given by $P_{i}(t)=\varepsilon_{0} \sum_{n} \chi_{i j}\left(\omega_{n}\right) \tilde{E}_{j}\left(\omega_{n}\right) e^{-\mathrm{i} \omega_{n} t}$, where $\chi_{i j}$ is the frequency-dependent linear susceptibility, for which $\chi_{i j}\left(\omega_{n}\right)=\chi_{i j}^{*}\left(-\omega_{n}\right)=\chi_{j i}^{*}\left(\omega_{n}\right)$. We accordingly rewrite the field energy density $U$ as

$$
U=\underbrace{\frac{1}{2} \varepsilon_{0} E_{i}(t) E_{i}(t)}_{H_{\text {vac }} / V_{\mathrm{c}}}+\underbrace{\frac{1}{2} P_{i}(t) E_{i}(t)}_{H_{\mathrm{phot}} / V_{\mathrm{c}}},
$$

where $H_{\text {vac }}$ is the Hamiltonian of the vacuum polarization, $H_{\text {phot }}$ that of the linear polarization of the medium, and $V_{\mathrm{c}}$ is the volume of the unit cell.

$\chi_{i j}$ is further dependent on the magnetic order and we expand it up to second order in the ferromagnetic and antiferromagnetic vectors, $\mathbf{m}=\mathbf{S}_{1}+\mathbf{S}_{2}$ and $\mathbf{I}=\mathbf{S}_{1}-\mathbf{S}_{2}$, respectively, as [8-11,21]

$$
\begin{aligned}
\chi_{i j}= & \chi_{i j}^{\mathrm{gs}}+\mathrm{i} \alpha_{i j k} m_{k}+\mathrm{i} \alpha_{i j k}^{\prime} l_{k} \\
& +\beta_{i j k o} m_{k} m_{o}+\beta_{i j k o}^{\prime} l_{k} l_{o}+\beta_{i j k o}^{\prime \prime} m_{k} l_{o},
\end{aligned}
$$

where $\chi_{i j}^{\mathrm{gs}}$ is the ground-state linear susceptibility, and $\alpha^{(\prime)}$ and $\beta^{(1, \prime)}$ are the frequency-dependent first and second order optomagnetic coefficients (or magnetic Raman tensors). The coupling of the laser pulse to the magnetization in first order is known as inverse Faraday effect, while the coupling in second order is known as inverse Cotton-Mouton effect [3-11,21].
For excitation energies below the band gap of insulating systems, the susceptibility mediates the Raman effect through virtual electronic states [51]. (The insulating system can, in principle, also be a Mott-insulating system [52]).

We assume that our model system is irradiated with light propagating along the $x$ direction, with electric-field components lying in the $y z$ plane, as illustrated in Fig. 2. For demonstration purposes, we choose one first-order and one second-order term to be nonzero and set $\alpha_{i j k}^{\prime}=\beta_{i j k o}=$ $\beta_{i j k o}^{\prime \prime}=0$. Note that in Refs. $[9,10]$ a similar geometry leads to vanishing terms linear in $l$, while the term scaling with $m^{2}$ can be neglected in antiferromagnets. For the remaining coefficients, $\alpha_{z y x}=-\alpha_{y z x}$ and $\beta_{z y y z}^{\prime}=\beta_{z y z y}^{\prime}=\beta_{y z z y}^{\prime}=\beta_{y z y z}^{\prime}$. In practice, the existence and magnitudes of the coefficients depends on material parameters and symmetries and can further vary with the dimensionality of the spin lattice. Using Eqs. (7) and (8), the interaction Hamiltonian of light with magnetic order reads

$$
\begin{aligned}
H_{\text {phot }}= & \frac{V_{c} \epsilon_{0}}{2}\left[\left(\chi_{y z}^{\mathrm{gs}}\left(\omega_{z}\right)-\mathrm{i} \alpha_{y z x}\left(\omega_{z}\right) m_{x}\right.\right. \\
& \left.\left.+2 \beta_{y z z y}^{\prime}\left(\omega_{z}\right) l_{y} l_{z}\right) \tilde{E}_{z}^{*}\left(\omega_{z}\right) e^{\mathrm{i} \omega_{z} t}+\text { c.c. }\right] E_{y}(t) \\
& +\frac{V_{c} \epsilon_{0}}{2}\left[\left(\chi_{y z}^{\mathrm{gs}}\left(\omega_{y}\right)+\mathrm{i} \alpha_{y z x}\left(\omega_{y}\right) m_{x}\right.\right. \\
& \left.\left.+2 \beta_{y z z y}^{\prime}\left(\omega_{y}\right) l_{y} l_{z}\right) \tilde{E}_{y}^{*}\left(\omega_{y}\right) e^{\mathrm{i} \omega_{y} t}+\text { c.c. }\right] E_{z}(t) .
\end{aligned}
$$

The effective optomagnetic field acting on the spin $\mathbf{S}_{s}$ is then given by $\mathbf{B}_{s}^{\text {eff }}=(\gamma \hbar)^{-1} \partial H_{\text {phot }} / \partial \mathbf{S}_{s}$.

To induce an effective optomagnetic field through the inverse Faraday effect, light traveling in the $x$ direction needs to be elliptically or circularly polarized, and we write for the $z$ and $y$ components of the electric field

$$
\begin{gathered}
E_{z}(t)=\mathcal{E}_{z}(t)\left(e^{-\mathrm{i} \omega_{z} t}+e^{\mathrm{i} \omega_{z} t}\right), \\
E_{y}(t)=\mathcal{E}_{y}(t)\left(\mathrm{i} e^{-\mathrm{i} \omega_{y} t}-\mathrm{i} e^{\mathrm{i} \omega_{y} t}\right) .
\end{gathered}
$$

Here, $\mathcal{E}_{i}(t)=\mathcal{E}_{i 0} \exp \left\{-t^{2} /\left[2(\tau / \sqrt{8 \ln 2})^{2}\right\}\right.$ is a Gaussian carrier envelope, $\mathcal{E}_{i 0}$ is the peak electric field along direction $i$, and $\tau$ is the full width at half maximum pulse duration. In general, $E_{z}(t)$ and $E_{y}(t)$ can have different center frequencies and their phase difference will change in time; in circularly polarized light, $\omega_{z}=\omega_{y}$ and their phase difference is $\pi / 4$.

Neglecting the dependence of $\chi_{i j}$ on the shape of the carrier envelope, assuming $l_{z}$ is constant in time as in Ref. [10], and replacing the spectral component $\tilde{E}_{i}$ with $\mathcal{E}_{i}$ in the time domain for a particular center frequency, we find that the inverse Faraday effect, mediated through the $\alpha_{z y x}$ term, generates an effective optomagnetic field, $\mathbf{B}_{s}^{\mathrm{IFE}}$, according to

$$
\begin{aligned}
\mathbf{B}_{s}^{\mathrm{IFE}}(t)= & \frac{\varepsilon_{0} V_{c}}{\gamma \hbar} \mathcal{E}_{z}(t) \mathcal{E}_{y}(t) \\
& \times\left[\alpha_{+} \cos \left(\omega_{-} t\right)+\alpha_{-} \cos \left(\omega_{+} t\right)\right] \hat{x},
\end{aligned}
$$

where we have used trigonometric product-to-sum and angle-sum identities, we defined $\omega_{ \pm}=\omega_{z} \pm \omega_{y}$ and $\alpha_{ \pm}=$ $\alpha_{z y x}\left(\omega_{y}\right) \pm \alpha_{z y x}\left(\omega_{z}\right)$, and where $\mathbf{B}_{1}^{\mathrm{IFE}}=\mathbf{B}_{2}^{\mathrm{IFE}}$. For circularly polarized light $\left(\mathcal{E}_{y}=\mathcal{E}_{z} \equiv \mathcal{E}\right.$ and $\left.\omega_{y}=\omega_{z}\right), \alpha_{-}$vanishes and we obtain the conventional equation for the inverse Faraday 
effect $[8,10,48]$,

$$
\mathbf{B}_{s}^{\mathrm{IFE}}(t)=\frac{\varepsilon_{0} V_{c}}{\gamma \hbar} \mathcal{E}^{2}(t) \alpha_{+} \hat{x} .
$$

The inverse Faraday effect involving circularly polarized light can therefore not produce sum-frequency components. More generally it can only produce sum-frequency components if $\alpha_{z y x}$ shows a large frequency dependence in order to make $\alpha_{-}$ significant. Conventionally, Raman tensors are only weakly frequency dependent in spectral ranges far below the band gap [23], and we expect similar behavior for magnetic Raman tensors here.

For an effective optomagnetic field induced by the inverse Cotton-Mouton effect, we assume a laser pulse traveling along the $x$ direction, which is linearly polarized in the $y z$ plane with an angle of $\phi$ with respect to the $z$ axis,

$$
\begin{aligned}
& E_{z}(t)=\mathcal{E}_{z}(t) \cos (\phi)\left(e^{-\mathrm{i} \omega_{z} t}+e^{\mathrm{i} \omega_{z} t}\right), \\
& E_{y}(t)=\mathcal{E}_{y}(t) \sin (\phi)\left(e^{-\mathrm{i} \omega_{y} t}+e^{\mathrm{i} \omega_{y} t}\right) .
\end{aligned}
$$

The effective optomagnetic field generated by the inverse Cotton-Mouton effect, $\mathbf{B}_{s}^{\mathrm{ICME}}$, mediated through the $\beta_{z y y z}^{\prime}$ term, then yields

$$
\begin{aligned}
\mathbf{B}_{1(2)}^{\mathrm{ICME}}(t)= & \pm \frac{\varepsilon_{0} V_{c}}{\gamma \hbar} \mathcal{E}_{z}(t) \mathcal{E}_{y}(t) \beta_{+}^{\prime} \sin (2 \phi) \\
& \times\left[\cos \left(\omega_{+} t\right)+\cos \left(\omega_{-} t\right)\right] l_{z} \hat{y},
\end{aligned}
$$

where we defined $\beta_{+}^{\prime}=\beta_{z y y z}^{\prime}\left(\omega_{y}\right)+\beta_{z y y z}^{\prime}\left(\omega_{z}\right)$. The inverse Cotton-Mouton effect is maximized when the linear polarization is oriented at 45 degrees between the $y$ and $z$ axes. Both the difference- and sum-frequency components scale identically with $\beta_{+}^{\prime}$, in contrast to the inverse Faraday effect.

\section{Effective phonomagnetic fields}

Next, we derive the effective phonomagnetic fields produced by coherent infrared-active phonons analogously to the previous section. In previous work, Ref. [21], fast-oscillating components were eliminated in the derivation of the Raman scattering mechanisms. In order to include both differenceand sum-frequency components, we write the interaction Hamiltonian, $H_{\text {phon }}$, as

$$
H_{\text {phon }}=\frac{1}{2} I_{i}(t) Q_{i}(t),
$$

where $Q_{i}(t)=\sum_{n} \tilde{Q}_{i}\left(\Omega_{n}\right) e^{-\mathrm{i} \Omega_{n} t}$ is the phonon amplitude and $I_{i}(t)=\sum_{n} D_{i j}\left(\Omega_{n}\right) \tilde{Q}_{j}\left(\Omega_{n}\right) e^{-\mathrm{i} \Omega_{n} t}$ can be regarded as phononic displacement field. We again use the Einstein notation for summing indices, which here denote the phonon band number and not spatial coordinates. $D_{i j}=\mathbf{q}_{i}^{\mathrm{T}} D \mathbf{q}_{j}$ is the projected dynamical matrix, where $\mathbf{q}_{i}$ is the eigenvector of phonon mode $i$ and $D$ the dynamical matrix. For $i=j$, we obtain the eigenfrequency of phonon mode $i$ as $\Omega_{i}=\sqrt{D_{i i}}$. We expand $D_{i j}$ to second order in the ferro- and antiferromagnetic vectors, $\mathbf{m}$ and $\mathbf{l}$, analogously to the linear susceptibility in the previous section, yielding [21]

$$
\begin{aligned}
D_{i j}= & D_{i j}^{\mathrm{gs}}+\mathrm{i} a_{i j k} m_{k}+\mathrm{i} a_{i j k}^{\prime} l_{k} \\
& +b_{i j k o} m_{k} m_{o}+b_{i j k o}^{\prime} l_{k} l_{o}+b_{i j k o}^{\prime \prime} m_{k} l_{o},
\end{aligned}
$$

where $D_{i j}^{\mathrm{gs}}$ is the projected dynamical matrix of the ground state, and $a^{(\prime)}$ and $b^{(\prime, \prime)}$ are the frequency-dependent first and second order magnetophononic coefficients (or magnetic ionic Raman tensors). The coupling of coherent infraredactive phonons to the magnetization in first order has been described as phonon inverse Faraday effect, and the coupling in second order as phonon inverse Cotton-Mouton effect [21,53].

In our model, we assume that the terms remaining in the expansion of the projected dynamical matrix are analogous to those in the expansion of the susceptibility for the optomagnetic effects, setting $a_{i j k}^{\prime}=b_{i j k o}=b_{i j k o}^{\prime \prime}=0$. For the remaining coefficients, $a_{z y x}=-a_{z y x}$ and $b_{z y y z}^{\prime}=b_{z y z y}^{\prime}=$ $b_{y z z y}^{\prime}=b_{y z y z}^{\prime}$. Using Eqs. (17) and (18), we obtain for the interaction Hamiltonian with coherent infrared-active phonons

$$
\begin{aligned}
H_{\mathrm{phon}}= & \frac{V_{c}}{2}\left[\left(D_{y z}^{\mathrm{gs}}\left(\Omega_{z}\right)-\mathrm{i} a_{y z x}\left(\Omega_{z}\right) m_{x}\right.\right. \\
& \left.\left.+2 b_{y z z y}^{\prime}\left(\Omega_{z}\right) l_{y} l_{z}\right) \tilde{Q}_{z}^{*}\left(\Omega_{z}\right) e^{i \Omega_{z} t}+\text { c.c. }\right] Q_{y}(t) \\
& +\frac{V_{c}}{2}\left[\left(D_{y z}^{\mathrm{gs}}\left(\Omega_{y}\right)+\mathrm{i} a_{y z x}\left(\Omega_{y}\right) m_{x}\right.\right. \\
& \left.\left.+2 b_{y z z y}^{\prime}\left(\Omega_{y}\right) l_{y} l_{z}\right) \tilde{Q}_{y}^{*}\left(\Omega_{y}\right) e^{i \Omega_{y} t}+\text { c.c. }\right] Q_{z}(t) .
\end{aligned}
$$

The effective phonomagnetic field acting on the $\operatorname{spin} \mathbf{S}_{s}$ is then given by $\mathbf{B}_{s}^{\text {eff }}=(\gamma \hbar)^{-1} \partial H_{\text {phon }} / \partial \mathbf{S}_{s}$.

To induce an effective phonomagnetic field through the phonon inverse Faraday effect, the coherent phonons have to be elliptically or circularly polarized, for example as a superposition of two phonons with orthogonal polarizations along $z$ and $y$ directions and frequencies $\Omega_{z}$ and $\Omega_{y}$, which are excited coherently by an elliptically or circularly polarized laser pulse traveling in $x$ direction. Without loss of generality, the phonon amplitudes can be written as

$$
\begin{gathered}
Q_{z}(t)=\mathcal{Q}_{z}(t)\left(e^{-\mathrm{i} \Omega_{z} t}+e^{\mathrm{i} \Omega_{z} t}\right), \\
Q_{y}(t)=\mathcal{Q}_{y}(t)\left(\mathrm{i} e^{-\mathrm{i} \Omega_{y} t}-\mathrm{i} e^{\mathrm{i} \Omega_{y} t}\right),
\end{gathered}
$$

where $\mathcal{Q}_{i}(t)$ are the envelopes of the coherently excited phonons that can be obtained by solving the phonon equations of motion. We assume that the projected dynamical matrix is constant during the entire time evolution and replace the spectral component $\tilde{Q}_{i}$ with $\mathcal{Q}_{i}$ in the time domain for a particular eigenfrequency. The effective phonomagnetic field generated by the phonon inverse Faraday effect, $\mathbf{B}_{s}^{\text {PIFE }}$, through the $a_{y z x}$ term then yields

$$
\begin{aligned}
\mathbf{B}_{s}^{\mathrm{PIFE}}= & \frac{V_{c}}{\gamma \hbar} \mathcal{Q}_{z}(t) \mathcal{Q}_{y}(t) \\
& \times\left[a_{+} \cos \left(\Omega_{-} t\right)+a_{-} \cos \left(\Omega_{+} t\right)\right] \hat{x},
\end{aligned}
$$

where we have again used trigonometric product-to-sum and angle-sum identities, defined $\Omega_{ \pm}=\Omega_{z} \pm \Omega_{y}$ and $a_{ \pm}=$ $a_{z y x}\left(\Omega_{y}\right) \pm a_{z y x}\left(\Omega_{z}\right)$, and where $\mathbf{B}_{1}^{\mathrm{PIFE}}=\mathbf{B}_{2}^{\mathrm{PIFE}} . \mathbf{B}_{s}^{\mathrm{PIFE}}$ can in short be written as cross product and in terms of the angular momentum of the phonons, $\mathbf{Q} \times \dot{\mathbf{Q}}[20,21,53-$ 59], so that $\mathbf{B}_{s}^{\mathrm{PIFE}}=a_{z y x} \mathbf{Q} \times \mathbf{Q}^{*} \equiv \tilde{a}_{z y x} \mathbf{Q} \times \dot{\mathbf{Q}}$, where $\mathbf{Q}=$ $\left(Q_{z}, Q_{y}, 0\right)$ and $a_{y z x}\left(\Omega_{i}\right)=\tilde{a}_{y z x} \Omega_{i}[21,53]$. The prefactors to the difference- and sum-frequency components can therefore be written as $a_{ \pm}=\tilde{a}_{y z x} \Omega_{ \pm} / 2$. For circularly polarized 
phonons (setting $\mathcal{Q}_{z}=\mathcal{Q}_{y} \equiv \mathcal{Q}, \Omega_{z}=\Omega_{y} \equiv \Omega$, and $\tilde{a}_{z y x} \equiv \tilde{a}$ ) we obtain the regular difference-frequency equation for the effective phonomagnetic field [20,21,53,58],

$$
\mathbf{B}_{s}^{\mathrm{PIFE}}=\frac{V_{c}}{\gamma \hbar} \mathcal{Q}^{2}(t) \Omega \tilde{a} \hat{x} .
$$

The phonon inverse Faraday effect, analogously to the inverse Faraday effect, produces no sum-frequency components in the case of circularly polarized phonons, in which $a_{-}=$ $\tilde{a}_{y z x} \Omega_{-} / 2=0$. A significant sum-frequency contribution is possible for strongly elliptical phonons with different frequencies for $z$ and $y$ polarizations.

For an effective phonomagnetic field induced by the phonon inverse Cotton-Mouton effect, we assume that two phonons with orthogonal polarizations $z$ and $y$ are excited in-phase,

$$
\begin{aligned}
& Q_{z}(t)=\mathcal{Q}_{z}(t) \cos (\phi)\left(e^{-\mathrm{i} \Omega_{z} t}+e^{\mathrm{i} \Omega_{z} t}\right), \\
& Q_{y}(t)=\mathcal{Q}_{y}(t) \sin (\phi)\left(e^{-\mathrm{i} \Omega_{y} t}+e^{\mathrm{i} \Omega_{y} t}\right),
\end{aligned}
$$

where $\phi$ is the angle of linear polarization with respect to the $z$ axis. The effective phonomagnetic fields, $\mathbf{B}_{s}^{\text {PICME }}$, mediated through the $b_{z y y z}^{\prime}$ term as the only contribution, yield

$$
\begin{aligned}
\mathbf{B}_{1(2)}^{\mathrm{PICME}}= & \pm \frac{V_{c}}{\gamma \hbar} \mathcal{Q}_{z}(t) \mathcal{Q}_{y}(t) b_{+} \sin (2 \phi) \\
& \times\left[\cos \left(\Omega_{+} t\right)+\cos \left(\Omega_{-} t\right)\right] l_{z} \hat{y}
\end{aligned}
$$

where we defined $b_{+}=b_{z y y z}^{\prime}\left(\Omega_{y}\right)+b_{z y y z}^{\prime}\left(\Omega_{z}\right)$. Analogously to the optomagnetic case, both difference- and sum-frequency components in the phonon inverse Cotton-Mouton effect scale identically with $b_{+}$and the effect is maximized when the polarization of the phonon modes is oriented at a 45 degree angle between the $z$ and $y$ axes.

To evaluate the effective phonomagnetic fields in Eqs. (22) and (26), we calculate the phonon amplitudes $Q_{i}$ by numerically solving the equations of motion

$$
\ddot{Q}_{i}+2 \kappa_{i} \dot{Q}_{i}+\Omega_{i}^{2} Q_{i}=Z_{i} E_{i}(t),
$$

where $\kappa_{i}$ are the phonon linewidths and $Z_{i}$ are the mode effective charges $[17,23,60]$. The shapes of the electric-field components $E_{i}$ for the excitation of phonons for the phonon inverse Faraday and phonon inverse Cotton-Mouton effects are given by Eqs. (10), (11), (14), and (15), respectively, where the center frequencies are chosen resonant with the phonon frequencies, $\omega_{i}=\Omega_{i}$.

The phonon amplitudes obtained by Eq. (27) serve as input to Eqs. (22) and (26) and consequently to Eq. (1). In this model, the phonons experience no feedback from the spin system and therefore act analogously to a nondepleting optical pulse. The feedback from a single Raman-active phonon into an infrared-active one through Raman-type coupling can lead to a beating signal of the amplitude but does not significantly contribute to the damping of the infrared-active phonon, which is primarily determined by the decay into acoustic phonons, and we expect the same to be true for magnons $[23,61]$. Note that while the coherently excited infrared-active phonons mediate their energy to the spins, they couple nonlinearly to other vibrational degrees of freedom and participate in ionic Raman scattering or two-phonon absorption by other phonons and may induce transient distortions in the crystal structure that alter the magnetic order quasistatically [62-70]. These effects and other non-Raman spin-phonon interactions [64,71-80], as well as two-magnon processes [81,82] and phenomena arising from Floquet driving [83-85], are not discussed in this work.

\section{NUMERICAL SPIN-DYNAMICS SIMULATIONS}

\section{A. Model parameters}

We turn to the numerical evaluations of the spin dynamics that the different optical and phononic drives induce according to the Landau-Lifshitz-Gilbert formalism in Eq. (1) and in the pump-probe geometry shown in Fig. 2. We use two different sets of anisotropy parameters for the antiferromagnetic Heisenberg model described by Eq. (3) in order to create one system with a high-frequency and one system with a low-frequency in-plane magnon mode. For the antiferromagnetic exchange coupling, we set $J=6 J_{\mathrm{NN}}=106 \mathrm{meV}$. We set $D_{x}=4.3 \mathrm{meV}$ and $D_{y}=1.0 \mathrm{meV}$ to get an in-plane magnon frequency of $5 \mathrm{THz}$, and $D_{x}=0.43 \mathrm{meV}$ and $D_{y}=$ $0.040 \mathrm{meV}$ to get an in-plane magnon frequency of $1 \mathrm{THz}$. In some materials, magnetic anisotropy induces structural distortions along the corresponding lattice directions, which shifts the frequency of the phonons polarized in this direction. As these distortions are generally very small we will neglect the influence of the magnetic anisotropy on the phonon frequencies here. We set the phenomenological damping of the spin precession to $\Gamma=2.4 \times 10^{-4}$ and the opto- and phonomagnetic coefficients to $\alpha_{z y x}=2.8 \times 10^{-58}, \beta_{z y y z}^{\prime}=2.3 \times 10^{-60}$, $a_{z y x}=4.9 \times 10^{-6} \mathrm{~m}^{3} \mathrm{~s}^{-2}, b_{z y y z}^{\prime}=2.2 \times 10^{-8} \mathrm{~m}^{3} \mathrm{~s}^{-2}$ in the unit system used here. In a real system, the opto- and phonomagnetic coefficients are strongly material dependent and have to be computed using first-principles calculations in order to produce quantitatively accurate efficiencies of the difference- and sum-frequency mechanisms [21]. We will therefore compare the difference- and sum-frequency mechanisms relative to each other in order to make the results independent of the absolute magnitudes of the coefficients.

The spin precession induced by the opto- and phonomagnetic fields can be detected through Faraday-rotation experiments, in which the Faraday rotation $\theta$ is proportional to the oscillation of the ferromagnetic component $\mathbf{m}$,

$$
\theta(t)=C \hat{x} \cdot \mathbf{m}(t),
$$

where the proportionality constant includes the gyromagnetic ratio $\gamma$, the ion density of the material, and the Verdet constant [30]. As we compare relative magnitudes of the spin precession, we set $C=1$ without loss of generality.

\section{B. Optomagnetic effects}

We now compare the relative efficiencies of the differenceand sum-frequency excitation mechanisms via the inverse Faraday and inverse Cotton-Mouton effects for the $5 \mathrm{THz}$ and $1 \mathrm{THz}$ magnon systems. For the laser pulses in Eqs. (10), (11), (14), and (15), we assume equal center frequencies and peak electric fields for the $z$ and $y$ components, $\omega_{z}=\omega_{y} \equiv \omega_{0}$ and $\mathcal{E}_{z 0}=\mathcal{E}_{y 0} \equiv \mathcal{E}_{0}$. For the difference-frequency components in impulsive stimulated Raman scattering, we use typical pulse 
Inverse Faraday effect

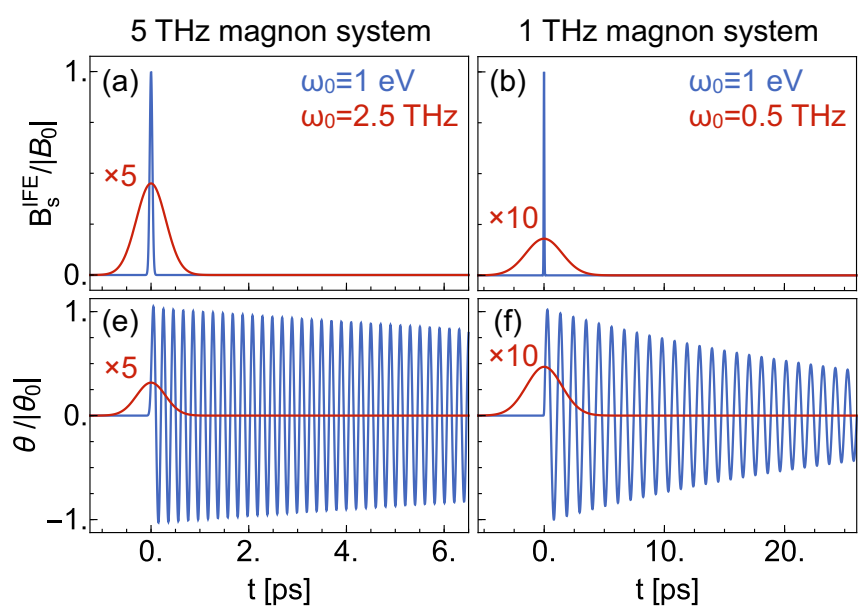

Inverse Cotton-Mouton effect

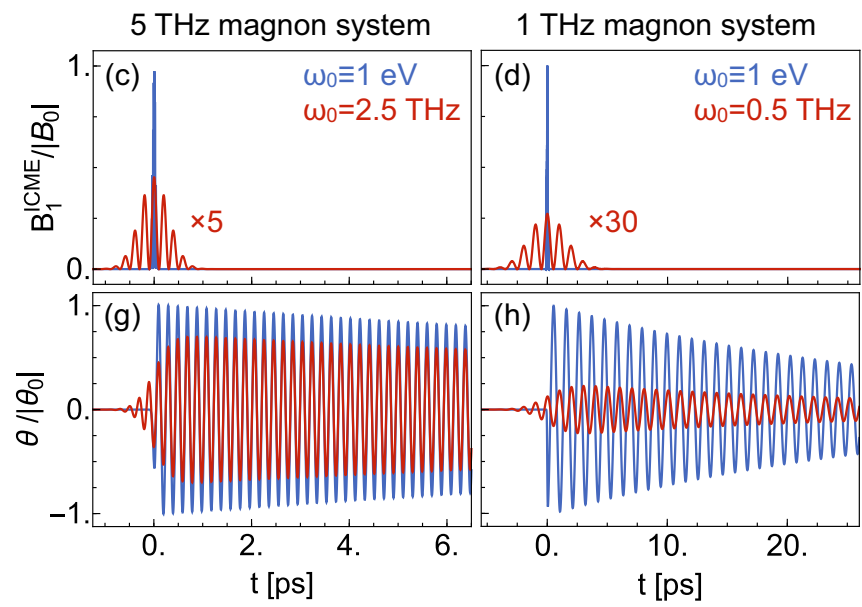

FIG. 3. Spin dynamics induced by the inverse Faraday and inverse Cotton-Mouton effects. In (a)-(d), we compare the effective magnetic fields, $B_{s}^{\mathrm{IFE}}$ and $B_{1}^{\mathrm{ICME}}$, produced by the different optical drives in the near-infrared $\left(\omega_{0} \equiv 1 \mathrm{eV}, \tau=90 \mathrm{fs}\right)$ and terahertz $\left(\omega_{0}=2.5 \mathrm{THz}, \tau=1\right.$ ps and $\omega_{0} \equiv 0.5 \mathrm{THz}, \tau=5 \mathrm{ps}$ ) spectral ranges in the $5 \mathrm{THz}$ and $1 \mathrm{THz}$ magnon systems. With these parameters, all three pulses have the same total energy. In (e)-(h) we compare the Faraday rotations $\theta$ around the $x$ direction arising from the spin dynamics induced by the effective magnetic fields. The Faraday rotations are directly proportional to the induced spin-precession amplitudes, $\theta(t) \propto \mathbf{m}(t)$, see Eq. (28). Both the effective magnetic fields and the rotations are normalized to those generated by the respective high-frequency drives, $B_{0}$ and $\theta_{0}$.

parameters in the near-infrared regime, with a photon energy of $1 \mathrm{eV}$ (center frequency $\omega_{0}=237 \mathrm{THz}$ ), full width at half maximum pulse duration of $\tau=90 \mathrm{fs}$, and a peak electric field of $\mathcal{E}_{0}=25 \mathrm{MV} / \mathrm{cm}$ [10]. For the sum-frequency components in two-photon absorption, we require pulses with center frequencies of half the magnon frequency, $\omega_{0}=\Omega_{\mathrm{IP}} / 2$. We therefore set the parameters to $\omega_{0}=2.5 \mathrm{THz}, \tau=1 \mathrm{ps}$, and $\mathcal{E}_{0}=7.5 \mathrm{MV} / \mathrm{cm}$ for the $5 \mathrm{THz}$ magnon system, and $\omega_{0}=0.5 \mathrm{THz}, \tau=5 \mathrm{ps}$, and $\mathcal{E}_{0}=3.4 \mathrm{MV} / \mathrm{cm}$ for the $1 \mathrm{THz}$ magnon system, where we chose the pulse durations and peak electric fields such that all three pulses have the same total energy.

We show the normalized effective optomagnetic fields produced by the inverse Faraday effect according to Eq. (12) and by the inverse Cotton-Mouton effect according to Eq. (16) in Figs. 3(a)-3(d), as well as the normalized Faraday rotations according to Eqs. (28) and (1) in Figs. 3(e)-3(h). The values of the optomagnetic fields and Faraday rotations are normalized to the maximum amplitudes of the respective high-frequency drives. In the inverse Faraday effect, only static responses of the effective optomagnetic fields $\mathbf{B}_{s}^{\mathrm{IFE}}$, resulting from difference-frequency components from both the near-infrared and terahertz pulses, show up. While the $1 \mathrm{eV}$ pulse is short enough to impulsively excite coherent spin precession, the $0.5 \mathrm{THz}$ pulse simply drags the ferromagnetic spin component along for the duration of the pulse. In the inverse Cotton-Mouton effect in contrast, an oscillatory component of the effective optomagnetic field $\mathbf{B}_{1}^{\mathrm{ICME}}$ is clearly visible. There, the $1 \mathrm{eV}$ as well as both terahertz pulses excite coherent spin precessions with similar magnitudes. The sum-frequency excitations by the terahertz pulses are not impulsive in nature and the spin-precession amplitude builds up gradually, compared to the sudden onset of the amplitude in the difference-frequency case. This behavior of the magnons in the impulsive stimulated Raman scattering and two-photon absorption processes underlying the inverse
Cotton-Mouton effect is equivalent to that of Raman-active phonons [23].

\section{Phonomagnetic effects}

We next compare the relative efficiencies of the differenceand sum-frequency excitation mechanisms via the phonon inverse Faraday and phonon inverse Cotton-Mouton effects for the $5 \mathrm{THz}$ and $1 \mathrm{THz}$ magnon systems. In a real material, the coherent excitation of infrared-active phonons imposes an additional limitation on the possible excitation strength of the laser pulses. When the amplitude of atomic motion exceeds a certain threshold, the structure of the crystal will break down. It is therefore more meaningful to compare the phonomagnetic effects in terms of equal phonon amplitudes and not in terms of equal total pulse energies as in the case of the optomagnetic effects.

For the difference-frequency components in ionic Raman scattering, we assume phonons polarized along the $z$ and $y$ directions with equal eigenfrequencies of $\Omega_{z}=\Omega_{y} \equiv \Omega_{\mathrm{IR}}=$ $15 \mathrm{THz}$, which are resonantly driven by a pulse with a center frequency of $\omega_{0}=\Omega_{\mathrm{IR}}$, a full width at half maximum pulse duration of $\tau=0.1 \mathrm{ps}$, and a peak electric field of $\mathcal{E}_{0}=23.7 \mathrm{MV} / \mathrm{cm}$. For the sum-frequency components in two-phonon absorption, the phonon frequencies have to be half the magnon frequency, $\Omega_{\mathrm{IR}}=\Omega_{\mathrm{IP}} / 2$, at $2.5 \mathrm{THz}$ for the $5 \mathrm{THz}$ magnon system and at $0.5 \mathrm{THz}$ for the $1 \mathrm{THz}$ magnon system. We yield phonon amplitudes equal to that of the $15 \mathrm{THz}$ phonon when we set the parameters as follows: $\omega_{0}=\Omega_{\mathrm{IR}}=2.5 \mathrm{THz}, \tau=1 \mathrm{ps}$, and $\mathcal{E}_{0}=0.4 \mathrm{MV} / \mathrm{cm}$ for the $5 \mathrm{THz}$ magnon system, and $\omega_{0}=\Omega_{\mathrm{IR}}=0.5 \mathrm{THz}, \tau=5 \mathrm{ps}$, and $\mathcal{E}_{0}=16 \mathrm{kV} / \mathrm{cm}$ for the $1 \mathrm{THz}$ magnon system. The excitation of the $2.5 \mathrm{THz}$ phonon with equal amplitude as the $15 \mathrm{THz}$ phonon therefore requires a factor of 350 less total pulse energy $\propto \mathcal{E}_{0}^{2} \tau$ and even a factor of 40000 less for the $0.5 \mathrm{THz}$ phonon. For the linewidths of all phonons, we use 
Phonon inverse Faraday effect

$5 \mathrm{THz}$ magnon system
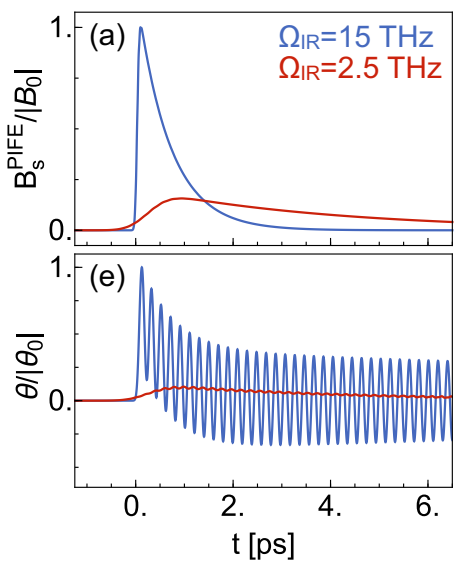

$1 \mathrm{THz}$ magnon system
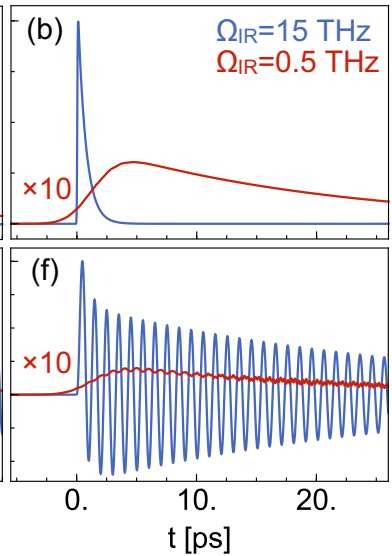

Phonon inverse Cotton-Mouton effect

$5 \mathrm{THz}$ magnon system $1 \mathrm{THz}$ magnon system
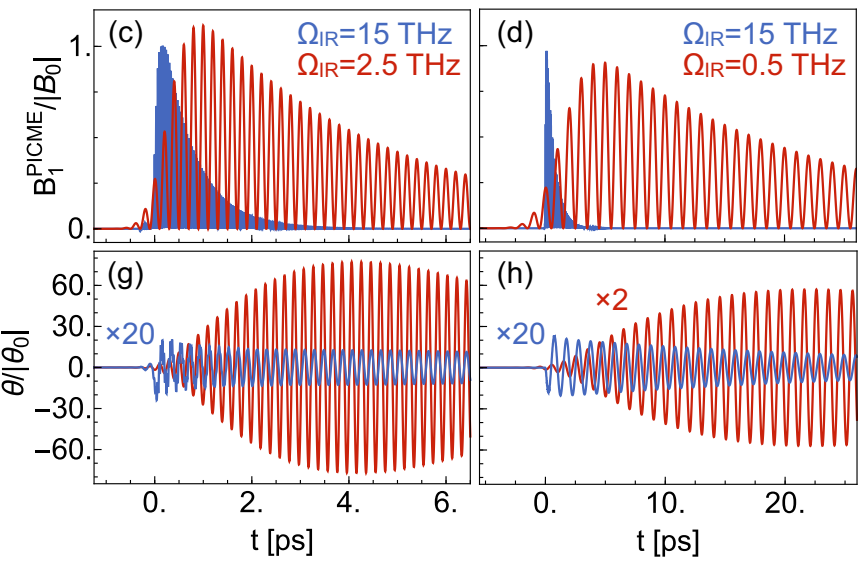

FIG. 4. Spin dynamics induced by the phonon inverse Faraday and phonon inverse Cotton-Mouton effects. In (a)-(d), we compare the effective magnetic fields, $B_{s}^{\text {PIFE }}$ and $B_{1}^{\text {PICME }}$, produced by the different phonons $(15,2.5$, and $0.5 \mathrm{THz})$ in the $5 \mathrm{THz}$ and $1 \mathrm{THz}$ magnon systems. In (e)-(h) we compare the Faraday rotations $\theta$ around the $x$ direction arising from the spin dynamics induced by the effective phonomagnetic fields. The Faraday rotations are directly proportional to the induced spin-precession amplitudes, $\theta(t) \propto \mathbf{m}(t)$, see Eq. (28). Both the effective magnetic fields and the rotations are normalized to those generated by the respective high-frequency drives, $B_{0}$ and $\theta_{0}$.

phenomenological values of $\kappa_{z}=\kappa_{y} \equiv \kappa_{\mathrm{IR}}=0.05 \times \Omega_{\mathrm{IR}}$. We further set the mode effective charges to $Z_{\mathrm{IR}}=1 e \AA$, where $e$ is the elementary charge [23].

We show the amplitudes of the coherently excited infraredactive phonons $Q_{z}$ according to Eq. (27) in Figs. 5(a) and 5(b). In the case of circular excitation, $Q_{y}$ (not shown in the figures) is shifted in phase by $\pi / 4$ with respect to $Q_{z}$ and in the case of linear excitation it is in phase with $Q_{z}$. We further show the normalized effective phonomagnetic fields produced by the phonon inverse Faraday effect according to Eq. (22) and by the phonon inverse Cotton-Mouton effect according to Eq. (26) in Figs. 4(a)-4(d), as well as the normalized Faraday rotations according to Eqs. (28) and (1) in Figs. 4(e)-4(h). The values of the phonomagnetic fields and Faraday rotations are normalized to the maximum amplitudes of the respective high-frequency drives.

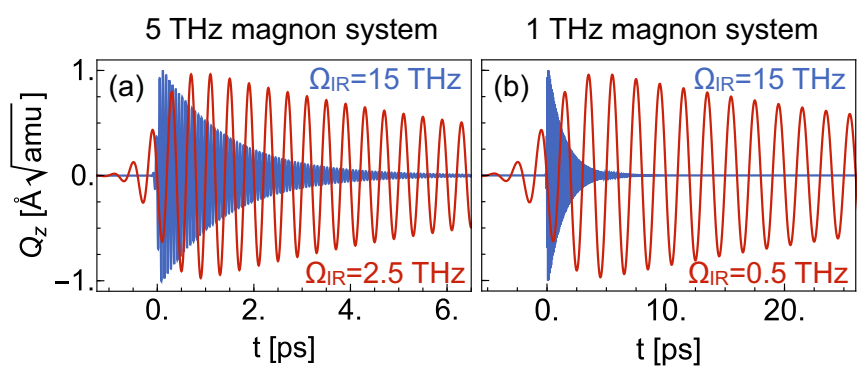

FIG. 5. Time evolution of the phonon amplitudes $Q_{z}$ in response to the excitations by resonant terahertz pulses. (a) 15 and $2.5 \mathrm{THz}$ phonons in the $5 \mathrm{THz}$ magnon system, and (b) 15 and $0.5 \mathrm{THz}$ phonons in the $1 \mathrm{THz}$ magnon system. We chose the pulse energies such that the maxima of $Q_{z}$ are equal for the high- and low-frequency phonons, respectively. In the case of circular excitation, $Q_{y}$ (not shown) is shifted in phase by $\pi / 4$ with respect to $Q_{z}$ and in the case of linear excitation it is in phase with $Q_{z}$.
The phonon inverse Faraday effect displays only static responses of the effective phonomagnetic fields $\mathbf{B}_{s}^{\mathrm{PIFE}}$, resulting from difference-frequency components from the 15 , 2.5 , and $0.5 \mathrm{THz}$ phonons, analogously to its optomagnetic counterpart. Analogously to the optomagnetic case, the highfrequency drive of the $15 \mathrm{THz}$ phonon leads to an impulsive excitation of the magnon through ionic Raman scattering, while the 2.5 and $0.5 \mathrm{THz}$ phonons induce a transient magnetization instead, as the ferromagnetic component $\mathbf{m}$ follows the envelope of the respective phonon. Notably, the $15 \mathrm{THz}$ phonon also induces a transient magnetization that is reminiscent of the transient structural distortion generated through nonlinear phonon-phonon coupling in nonlinear phononics experiments [15-18].

For the phonon inverse Cotton-Mouton effect, a strong oscillatory component is visible in the effective phonomagnetic field $\mathbf{B}_{1}^{\mathrm{PICME}}$. While all three phonons are excited with similar amplitudes and yield similar magnitudes of effective magnetic fields, the spin-precession amplitudes and therefore Faraday rotations induced by the 2.5 and $0.5 \mathrm{THz}$ phonons exceed those of the $15 \mathrm{THz}$ phonon by a factor of 30-50. This is due to the nonimpulsive nature of the sumfrequency process that gradually builds up the amplitude of the magnon, and which benefits from the long lifetime of the low-frequency phonons, while the impulsive excitation through the difference-frequency components is independent of the envelope of the high-frequency phonon. Analog to the optomagnetic case, the behavior of the magnons in the ionic Raman scattering and two-phonon absorption processes underlying the phonon inverse Cotton-Mouton effect is similar to that of Raman-active phonons [23].

\section{DISCUSSION}

Our results show that the inverse Faraday and phonon inverse Faraday effects do not produce sum-frequency 
components for circularly polarized light or phonons, respectively. The weak dependence of the first-order optomagnetic coefficients on the frequency of light and phonons makes it further unlikely that significant excitation will be achieved through elliptical polarization. Intriguingly however, the difference-frequency components of the phonon inverse Faraday effect induce a transient magnetization that could be considered a magnetic analog of the transient structural distortion in nonlinear phononics [15-18,60,62-67,69,70,86].

The inverse Cotton-Mouton and phonon inverse CottonMouton effects in contrast produce strong sum-frequency components that scale identically in terms of the second-order opto- and phonomagnetic coefficients as the differencefrequency components. Here, for the same total pulse energies and magnitudes of the optomagnetic coefficients, similar spinprecession amplitudes can be achieved through impulsive stimulated Raman scattering and two-photon absorption. In the phonomagnetic effects, spin-precession amplitudes induced by two-phonon absorption are at least an order of magnitude larger than those induced by ionic Raman scattering for equally strong phonon excitations and phonomagnetic coefficients. This difference arises from the nonimpulsive nature of the sum-frequency mechanisms in combination with the long lifetimes of the low-frequency phonons, where the spin-precession amplitude can build up gradually over time. In addition, the pulse energies required to excite these lowfrequency phonons are orders of magnitude lower than the ones required to excite high-frequency phonons, making the phonon inverse Cotton-Mouton effect particularly efficient.

From a feasibility perspective, optomagnetic sumfrequency excitation can straightforwardly be implemented by choosing the center frequency of the laser pulse at half of the magnon frequency. The feasibility of the phonomagnetic sumfrequency mechanism in turn strongly depends on the material properties that determine the magnon and phonon frequencies. For low-frequency magnons, as the example of the $1 \mathrm{THz}$ magnon here shows, phonons with an eigenfrequency of 0.5 $\mathrm{THz}$ would be required that are not present in most existing compounds. The phonomagnetic sum-frequency mechanism should therefore be more applicable to high-frequency magnons (and possibly two-magnon excitations, which double the required excitation energy compared to one-magnon excitations), for example in complex magnetic structures such as yttrium iron garnet [87], which host a large number of magnon and phonon modes. There, the high selectivity provided by the sum-frequency mechanisms due to their resonant conditions in combination with the high selectivity of coherent phonon excitations achievable in recent years [88] may become powerful tools for spin dynamical control in the future.

\section{ACKNOWLEDGMENTS}

We acknowledge fruitful discussions with Sebastian Maehrlein, Alexandra Kalashnikova, Tobias Kampfrath, Christian Tzschaschel, and Tomáš Neuman. This work was supported by the Swiss National Science Foundation under Project No. 184259, the DARPA DRINQS program under Award No. D18AC00014, as well as by the Department of Energy Photonics at Thermodynamic Limits Energy Frontier Research Center under Grant No. DE-SC0019140. D.S.W. is supported by a NSF Graduate Research Fellowship. P.N. is a Moore Inventor Fellow through Grant No. GBMF8048 from the Gordon and Betty Moore Foundation.
[1] S. De Silvestri, J. G. Fujimoto, E. P. Ippen, E. B. Gamble Jr., L. R. Williams, and K. A. Nelson, Femtosecond Time-Resolved Measurements of Optic Phonon Dephasing by Impulsive Stimulated Raman Scattering in $\alpha$-Perylene Crystal from 20 to $300 \mathrm{~K}$, Chem. Phys. Lett. 116, 146 (1985).

[2] R. Merlin, Generating coherent THz phonons with light pulses, Solid State Commun. 102, 207 (1997).

[3] A. V. Kimel, A. Kirilyuk, P. A. Usachev, R. V. Pisarev, A. M. Balbashov, and Th. Rasing, Ultrafast non-thermal control of magnetization by instantaneous photomagnetic pulses, Nature (London) 435, 655 (2005).

[4] A. M. Kalashnikova, A. V. Kimel, R. V. Pisarev, V. N. Gridnev, A. Kirilyuk, and Th. Rasing, Impulsive Generation of Coherent Magnons by Linearly Polarized Light in the EasyPlane Antiferromagnet $\mathrm{FeBO}_{3}$, Phys. Rev. Lett. 99, 167205 (2007).

[5] A. M. Kalashnikova, A. V. Kimel, R. V. Pisarev, V. N. Gridnev, P. A. Usachev, A. Kirilyuk, and T. Rasing, Impulsive excitation of coherent magnons and phonons by subpicosecond laser pulses in the weak ferromagnet $\mathrm{FeBO}_{3}$, Phys. Rev. B 78, 104301 (2008).

[6] V. N. Gridnev, Phenomenological theory for coherent magnon generation through impulsive stimulated Raman scattering, Phys. Rev. B 77, 094426 (2008).
[7] D. Popova, A. Bringer, and S. Blügel, Theoretical investigation of the inverse Faraday effect via a stimulated Raman scattering process, Phys. Rev. B 85, 094419 (2012).

[8] A. M. Kalashnikova, A. V. Kimel, and R. V. Pisarev, Ultrafast opto-magnetism, Phys. Usp. 58, 969 (2015).

[9] T. Satoh, R. Iida, T. Higuchi, Y. Fujii, A. Koreeda, H. Ueda, T. Shimura, K. Kuroda, V. I. Butrim, and B. A. Ivanov, Excitation of coupled spin-orbit dynamics in cobalt oxide by femtosecond laser pulses, Nat. Commun. 8, 638 (2017).

[10] C. Tzschaschel, K. Otani, R. Iida, T. Shimura, H. Ueda, S. Günther, M. Fiebig, and T. Satoh, Ultrafast optical excitation of coherent magnons in antiferromagnetic NiO, Phys. Rev. B 95, 174407 (2017).

[11] P. Khan, M. Kanamaru, K. Matsumoto, T. Ito, and T. Satoh, Ultrafast light-driven simultaneous excitation of coherent terahertz magnons and phonons in multiferroic $\mathrm{BiFeO}_{3}$, Phys. Rev. B 101, 134413 (2020).

[12] A. A. Maradudin and R. F. Wallis, Ionic Raman Effect. I. Scattering by Localized Vibration Modes, Phys. Rev. B 2, 4294 (1970).

[13] R. F. Wallis and A. A. Maradurin, Ionic Raman Effect. II. The First-Order Ionic Raman Effect, Phys. Rev. B 3, 2063 (1971).

[14] L. B. Humphreys, Ionic Raman Effect. III. First- and SecondOrder Ionic Raman Effects, Phys. Rev. B 6, 3886 (1972). 
[15] M. Först, C. Manzoni, S. Kaiser, Y. Tomioka, Y. Tokura, R. Merlin, and A. Cavalleri, Nonlinear phononics as an ultrafast route to lattice control, Nat. Phys. 7, 854 (2011).

[16] M. Först, R. Mankowsky, H. Bromberger, D. M. Fritz, H. Lemke, D. Zhu, M. Chollet, Y. Tomioka, Y. Tokura, R. Merlin, J. P. Hill, S. L. Johnson, and A. Cavalleri, Displacive lattice excitation through nonlinear phononics viewed by femtosecond X-ray diffraction, Solid State Commun. 169, 24 (2013).

[17] A. Subedi, A. Cavalleri, and A. Georges, Theory of nonlinear phononics for coherent light control of solids, Phys. Rev. B 89, 220301(R) (2014).

[18] R. Mankowsky, M. Först, T. Loew, J. Porras, B. Keimer, and A. Cavalleri, Coherent modulation of the $\mathrm{YBa}_{2} \mathrm{Cu}_{3} \mathrm{O}_{6+x}$ atomic structure by displacive stimulated ionic Raman scattering, Phys. Rev. B 91, 094308 (2015).

[19] T. F. Nova, A. Cartella, A. Cantaluppi, M. Först, D. Bossini, R. V. Mikhaylovskiy, A. V. Kimel, R. Merlin, and A. Cavalleri, An effective magnetic field from optically driven phonons, Nat. Phys. 13, 132 (2017).

[20] D. M. Juraschek, M. Fechner, A. V. Balatsky, and N. A. Spaldin, Dynamical Multiferroicity, Phys. Rev. Mater. 1, 014401 (2017).

[21] D. M. Juraschek, P. Narang, and N. A. Spaldin, Phonomagnetic analogs to opto-magnetic effects, Phys. Rev. Research 2, 043035 (2020).

[22] S. Maehrlein, A. Paarmann, M. Wolf, and T. Kampfrath, Terahertz Sum-Frequency Excitation of a Raman-Active Phonon, Phys. Rev. Lett. 119, 127402 (2017).

[23] D. M. Juraschek and S. F. Maehrlein, Sum-frequency ionic Raman scattering, Phys. Rev. B 97, 174302 (2018).

[24] B. E. Knighton, R. T. Hardy, C. L. Johnson, L. M. Rawlings, J. T. Woolley, C. Calderon, A. Urrea, and J. A. Johnson, Terahertz waveform considerations for nonlinearly driving lattice vibrations, J. Appl. Phys. 125, 144101 (2019).

[25] C. L. Johnson, B. E. Knighton, and J. A. Johnson, Distinguishing Nonlinear Terahertz Excitation Pathways with Two-Dimensional Spectroscopy, Phys. Rev. Lett. 122, 073901 (2019).

[26] A. A. Melnikov, K. N. Boldyrev, Yu. G. Selivanov, V. P. Martovitskii, S. V. Chekalin, and E. A. Ryabov, Coherent phonons in $\mathrm{B}_{2} \mathrm{Se}_{3}$ film generated by an intense single-cycle THz pulse, Phys. Rev. B 97, 214304 (2018).

[27] M. Kozina, M. Fechner, P. Marsik, T. Van Driel, J. M. Glownia, C. Bernhard, M. Radovic, S. Bonetti, U. Staub, and M. C. Hoffmann, Terahertz-Driven Phonon Upconversion in $\mathrm{SrTiO}_{3}$, Nat. Phys. 15, 387 (2019).

[28] A. A. Melnikov, Yu. G. Selivanov, and S. V. Chekalin, Dynamical splitting of $E_{g}$ phonon mode in a terahertz-pumped $\mathrm{Bi}_{2} \mathrm{Se}_{3}$ crystal, Phys. Rev. B 102, 224301 (2020).

[29] M. T. Hutchings and E. J. Samuelsen, Measurement of SpinWave Dispersion in $\mathrm{NiO}$ by Inelastic Neutron Scattering and Its Relation to Magnetic Properties, Phys. Rev. B 6, 3447 (1972).

[30] T. Kampfrath, A. Sell, G. Klatt, A. Pashkin, S. Mährlein, T. Dekorsy, M. Wolf, M. Fiebig, A. Leitenstorfer, and R. Huber, Coherent terahertz control of antiferromagnetic spin waves, Nat. Photonics 5, 31 (2011).

[31] S. M. Rezende, A. Azevedo, and R. L. Rodríguez-Suárez, Introduction to antiferromagnetic magnons, J. Appl. Phys. 126, 151101 (2019).

[32] S. M. Rezende and N. Zagury, Coherent magnon states, Phys. Lett. A 29, 47 (1969).
[33] S. M. Rezende, Fundamentals of Magnonics (Springer Nature, Switzerland, 2020).

[34] P. A. Fleury and R. Loudon, Scattering of light by one- and two-magnon excitations, Phys. Rev. 166, 514 (1968).

[35] M. Rini, R. Tobey, N. Dean, J. Itatani, Y. Tomioka, Y. Tokura, R. W. Schoenlein, and A. Cavalleri, Control of the electronic phase of a manganite by mode-selective vibrational excitation, Nature (London) 449, 72 (2007).

[36] R. I. Tobey, D. Prabhakaran, A. T. Boothroyd, and A. Cavalleri, Ultrafast Electronic Phase Transition in $\mathrm{La}_{1 / 2} \mathrm{Sr}_{3 / 2} \mathrm{MnO}_{4}$ by Coherent Vibrational Excitation: Evidence for Nonthermal Melting of Orbital Order, Phys. Rev. Lett. 101, 197404 (2008).

[37] P. Beaud, S. L. Johnson, E. Vorobeva, U. Staub, R. A. De Souza, C. J. Milne, Q. X. Jia, and G. Ingold, Ultrafast Structural Phase Transition Driven by Photoinduced Melting of Charge and Orbital Order, Phys. Rev. Lett. 103, 155702 (2009).

[38] A. D. Caviglia, M. Först, R. Scherwitzl, V. Khanna, H. Bromberger, R. Mankowsky, R. Singla, Y. D. Chuang, W. S. Lee, O. Krupin, W. F. Schlotter, J. J. Turner, G. L. Dakovski, M. P. Minitti, J. Robinson, V. Scagnoli, S. B. Wilkins, S. A. Cavill, M. Gibert, S. Gariglio, P. Zubko, J. M. Triscone, J. P. Hill, S. S. Dhesi, and A. Cavalleri, Photoinduced melting of magnetic order in the correlated electron insulator $\mathrm{NdNiO}_{3}$, Phys. Rev. B 88, 220401(R) (2013).

[39] W. Hu, S. Catalano, M. Gibert, J. M. Triscone, and A. Cavalleri, Broadband terahertz spectroscopy of the insulator-metal transition driven by coherent lattice deformation at the $\mathrm{SmNiO}_{3} / \mathrm{LaAlO}_{3}$ interface, Phys. Rev. B 93, 161107(R) (2016).

[40] M. Först, K. R. Beyerlein, R. Mankowsky, W. Hu, G. Mattoni, S. Catalano, M. Gibert, O. Yefanov, J. N. Clark, A. Frano, J. M. Glownia, M. Chollet, H. Lemke, B. Moser, S. P. Collins, S. S. Dhesi, A. D. Caviglia, J. M. Triscone, and A. Cavalleri, Multiple Supersonic Phase Fronts Launched at a Complex-Oxide Heterointerface, Phys. Rev. Lett. 118, 027401 (2017).

[41] A. H. M. Reid, A. V. Kimel, A. Kirilyuk, J. F. Gregg, and Th. Rasing, Investigation of the femtosecond inverse Faraday effect using paramagnetic $\mathrm{Dy}_{3} \mathrm{Al}_{5} \mathrm{O}_{12}$, Phys. Rev. B 81, 104404 (2010).

[42] R. V. Mikhaylovskiy, E. Hendry, and V. V. Kruglyak, Ultrafast inverse Faraday effect in a paramagnetic terbium gallium garnet crystal, Phys. Rev. B 86, 100405(R) (2012).

[43] S. D. Gorelov, E. A. Mashkovich, M. V. Tsarev, and M. I. Bakunov, Terahertz Cherenkov radiation from ultrafast magnetization in terbium gallium garnet, Phys. Rev. B 88, 220411(R) (2013).

[44] D. Popova, A. Bringer, and S. Blügel, Theory of the inverse Faraday effect in view of ultrafast magnetization experiments, Phys. Rev. B 84, 214421 (2011).

[45] M. Berritta, R. Mondal, K. Carva, and P. M. Oppeneer, Ab Initio Theory of Coherent Laser-Induced Magnetization in Metals, Phys. Rev. Lett. 117, 137203 (2016).

[46] A. H. Majedi and B. Lounis, Nonlinear Optics of Optomagnetics; Quantum and Classical Treatments, Phys. Rev. B 102, 214401 (2020).

[47] P. S. Pershan, Nonlinear optical properties of solids: energy considerations, Phys. Rev. 130, 919 (1963).

[48] P. S. Pershan, J. P. van der Ziel, and L. D. Malmstrom, Theoretical Discussion of the Inverse Faraday Effect, Raman Scattering, and Related Phenomena, Phys. Rev. 143, 574 (1966). 
[49] B. A. Zon and V. Y. Kupershmidt, Inverse Faraday effect in magnetically ordered crystals, Z. Eksp. Teor. Fiz. 84, 363 (1983).

[50] Robert F. Boyd, Nonlinear Optics (Academic Press, Inc., Amsterdam, Boston, 2008).

[51] Y. R. Shen and N. Bloembergen, Interaction between Light Waves and Spin Waves, Phys. Rev. 143, 372 (1964).

[52] B. S. Shastry and B. I. Shraiman, Theory of Raman Scattering in Mott-Hubbard Systems, Phys. Rev. Lett. 65, 1068 (1990).

[53] D. M. Juraschek and P. Narang, Giant phonon-induced effective magnetic fields in $4 f$ paramagnets, arXiv:2007.10556.

[54] L. Sheng, D. N. Sheng, and C. S. Ting, Theory of the Phonon Hall Effect in Paramagnetic Dielectrics, Phys. Rev. Lett. 96, 155901 (2006).

[55] L. Zhang and Q. Niu, Angular Momentum of Phonons and the Einstein-de Haas Effect, Phys. Rev. Lett. 112, 085503 (2014).

[56] D. A. Garanin and E. M. Chudnovsky, Angular momentum in spin-phonon processes, Phys. Rev. B 92, 024421 (2015).

[57] J. J. Nakane and H. Kohno, Angular momentum of phonons and its application to single-spin relaxation, Phys. Rev. B 97, 174403 (2018).

[58] D. M. Juraschek and N. A. Spaldin, Orbital magnetic moments of phonons, Phys. Rev. Materials 3, 064405 (2019).

[59] S. Streib, The difference between angular momentum and pseudo angular momentum, arXiv:2010.15616.

[60] M. Fechner and N. A. Spaldin, Effects of intense optical phonon pumping on the structure and electronic properties of yttrium barium copper oxide, Phys. Rev. B 94, 134307 (2016).

[61] D. M. Juraschek, T. Neuman, J. Flick, and P. Narang, Cavity control of nonlinear phononics, arXiv:1912.00122.

[62] D. M. Juraschek, M. Fechner, and N. A. Spaldin, Ultrafast Structure Switching through Nonlinear Phononics, Phys. Rev. Lett. 118, 054101 (2017).

[63] P. G. Radaelli, Breaking symmetry with light: Ultrafast ferroelectricity and magnetism from three-phonon coupling, Phys. Rev. B 97, 085145 (2018).

[64] M. Fechner, A. Sukhov, L. Chotorlishvili, C. Kenel, J. Berakdar, and N. A. Spaldin, Magnetophononics: ultrafast spin control through the lattice, Phys. Rev. Mater. 2, 064401 (2018).

[65] M. Gu and J. M. Rondinelli, Nonlinear phononic control and emergent magnetism in Mott insulating titanates, Phys. Rev. B 98, 024102 (2018).

[66] G. Khalsa and N. A. Benedek, Ultrafast optically induced ferromagnetic/anti-ferromagnetic phase transition in $\mathrm{GdTiO}_{3}$ from first principles, npj Quantum Mater. 3, 15 (2018).

[67] S. F. Maehrlein, I. Radu, P. Maldonado, A. Paarmann, M. Gensch, A. M. Kalashnikova, R. V. Pisarev, M. Wolf, P. M. Oppeneer, J. Barker, and T. Kampfrath, Dissecting spin-phonon equilibration in ferrimagnetic insulators by ultrafast lattice excitation, Sci. Adv. 4, eaar5164 (2018).

[68] D. Afanasiev, J. R. Hortensius, B. A. Ivanov, A. Sasani, E. Bousquet, Y. M. Blanter, R. V. Mikhaylovskiy, A. V. Kimel, and A. D. Caviglia, Ultrafast control of magnetic interactions via light-driven phonons, Nature Materials, Nat. Mater. (2021).

[69] A. S. Disa, M. Fechner, T. F. Nova, B. Liu, M. Först, D. Prabhakaran, P. G. Radaelli, and A. Cavalleri, Polarizing an antiferromagnet by optical engineering of the crystal field, Nat. Phys. 16, 937 (2020).

[70] M. Rodriguez-Vega, Z.-X. Lin, A. Leonardo, A. Ernst, G. Chaudhary, M. G. Vergniory, and G. A. Fiete, Phonon-mediated dimensional crossover in bilayer $\mathrm{CrI}_{3}$, Phys. Rev. B 102, 081117(R) (2020).

[71] J. Fransson, D. Thonig, P. F. Bessarab, S. Bhattacharjee, J. Hellsvik, and L. Nordström, Microscopic theory for coupled atomistic magnetization and lattice dynamics, Phys. Rev. Mater. 1, 074404 (2017).

[72] Y. Hashimoto, S. Daimon, R. Iguchi, Y. Oikawa, K. Shen, K. Sato, D. Bossini, Y. Tabuchi, T. Satoh, B. Hillebrands, G. E. W. Bauer, T. H. Johansen, A. Kirilyuk, T. Rasing, and E. Saitoh, All-optical observation and reconstruction of spin wave dispersion, Nat. Commun. 8, 15859 (2017).

[73] S. Roychoudhury and S. Sanvito, Spin-Phonon coupling parameters from maximally localized Wannier functions and first-principles electronic structure: Single-crystal durene, Phys. Rev. B 98, 125204 (2018).

[74] S. Streib, H. Keshtgar, and G. E. W. Bauer, Damping of Magnetization Dynamics by Phonon Pumping, Phys. Rev. Lett. 121, 027202 (2018).

[75] T. Nomura, X. X. Zhang, S. Zherlitsyn, J. Wosnitza, Y. Tokura, N. Nagaosa, and S. Seki, Phonon Magnetochiral Effect, Phys. Rev. Lett. 122, 145901 (2019).

[76] J. Hellsvik, D. Thonig, K. Modin, D. Iuşan, A. Bergman, O. Eriksson, L. Bergqvist, and A. Delin, General method for atomistic spin-lattice dynamics with first-principles accuracy, Phys. Rev. B 99, 104302 (2019).

[77] P. Maldonado and Y. O. Kvashnin, Microscopic theory of ultrafast out-of-equilibrium magnon-phonon dynamics in insulators, Phys. Rev. B 100, 014430 (2019).

[78] C. Berk, M. Jaris, W. Yang, S. Dhuey, S. Cabrini, and H. Schmidt, Strongly coupled magnon-phonon dynamics in a single nanomagnet, Nat. Commun. 10, 2652 (2019).

[79] S. Streib, N. Vidal-Silva, K. Shen, and G. E. W. Bauer, Magnonphonon interactions in magnetic insulators, Phys. Rev. B 99, 184442 (2019).

[80] A. Rückriegel, S. Streib, G. E. W. Bauer, and R. A. Duine, Angular momentum conservation and phonon spin in magnetic insulators, Phys. Rev. B 101, 104402 (2020).

[81] D. Bossini, S. Dal Conte, Y. Hashimoto, A. Secchi, R. V. Pisarev, Th Rasing, G. Cerullo, and A. V. Kimel, Macrospin dynamics in antiferromagnets triggered by sub-20 femtosecond injection of nanomagnons, Nat. Commun. 7, 10645 (2016).

[82] D. Bossini, S. Dal Conte, G. Cerullo, O. Gomonay, R. V. Pisarev, M. Borovsak, D. Mihailovic, J. Sinova, J. H. Mentink, Th Rasing, and A. V. Kimel, Laser-driven quantum magnonics and terahertz dynamics of the order parameter in antiferromagnets, Phys. Rev. B 100, 024428 (2019).

[83] S. A. Owerre, Floquet topological magnons, J. Phys. Commun. 1, 021002 (2017).

[84] Martin Claassen, Hong Chen Jiang, Brian Moritz, and Thomas P. Devereaux, Dynamical time-reversal symmetry breaking and photo-induced chiral spin liquids in frustrated Mott insulators, Nat. Commun. 8, 1192 (2017).

[85] E. Viñas Boström, M. Claassen, J. W. McIver, G. Jotzu, A. Rubio, and M. A. Sentef, Light-induced topological magnons in two-dimensional van der Waals magnets, SciPost Phys. 9, 061 (2020).

[86] A. Subedi, Proposal for ultrafast switching of ferroelectrics using midinfrared pulses, Phys. Rev. B 92, 214303 (2015). 
[87] A. J. Princep, R. A. Ewings, S. Ward, S. Tóth, C. Dubs, D. Prabhakaran, and A. T. Boothroyd, The full magnon spectrum of yttrium iron garnet, npj Quantum Mater. 2, 63 (2017).
[88] B. Liu, M. Först, M. Fechner, D. Nicoletti, J. Porras, T. Loew, B. Keimer, and A. Cavalleri, Pump Frequency Resonances for Light-Induced Incipient Superconductivity in $\mathrm{YBa}_{2} \mathrm{Cu}_{3} \mathrm{O}_{6.5}$, Phys. Rev. X 10, 011053 (2020). 